Discrete Comput Geom 26:321-351 (2001)

DOI: $10.1007 / \mathrm{s} 00454-001-0040-0$

\title{
Bounded Valence Excess and the Parabolicity of Tilings
}

\author{
A. Repp \\ P. O. Box 1779, Kea'au, HI 96749, USA \\ mahaloakua@aol.com
}

\begin{abstract}
The fat flow modulus is a discrete version of the classical conformal modulus; one can use it to classify triangulations of half-open annuli as parabolic or hyperbolic. There exist various criteria for parabolicity; several of these criteria involve the vertex valences of the triangulation. In this paper we decompose the half-open annulus into a family of concentric sub-annuli. We can estimate the fat flow moduli of these sub-annuli in terms of their vertex valences. By using the Layer Theorem of Cannon et al. [2], we sum the estimates for these sub-annuli to prove a conjecture of He and Schramm [6]. The result is a new parabolicity criterion.
\end{abstract}

\section{Combinatorial Modulus}

In 1989 Pansu had already used a version of conformal modulus to discriminate among spaces of negative curvature [7]. About the same time, Cannon was using a discrete version of conformal modulus to prove his Combinatorial Riemann Mapping Theorem; the paper appeared in 1994 [1]. Cannon et al. (see [2]-[5]), as well as He and Schramm [6] later employed the same concept in various ways.

The specific discrete modulus used by Cannon is the combinatorial fat flow modulus. The formulation of this modulus given below is essentially due to Cannon et al. [4].

We say that $X$ is a half-open annulus if it is homeomorphic to $\left\{(x, y) \mid 1 \leq x^{2}+y^{2}<\right.$ $2\}$. The homeomorphism $\psi$ will map $\partial X$ to the unit circle; we call this boundary the bottom of $X$. Now suppose $X$ is tiled by closed topological disks. We assume that the tiling is locally finite, but the number of tiles will be (countably) infinite. Let $w$ be a nonzero function that assigns to each tile $t$ in $X$ a nonnegative weight, $w(t)$. We assume that $w$ is $\ell^{2}$; i.e., $\sum_{t \in X} w(t)^{2}<\infty$. Then the $w$-area of $X$, denoted $A_{w}$, is the sum of the squares of the weights of the tiles. If $C$ is a collection of tiles, then the $w$-length of $C$, denoted $\operatorname{len}_{w}(C)$, is the sum of $w(t)$, taken over the tiles $t$ in $C$.

We now define fat flows and skinny cuts. Let $f$ be a connected set of tiles in $X$, and suppose there exists a topological path $\alpha:[0,1) \rightarrow X$ such that the tiles in $f$ cover $\alpha$. 
Furthermore, suppose $f$ includes all tiles which intersect $\alpha$. For any $r \in[0,1)$, let $F(r)$ be the union of all tiles in $f$ which intersect $\alpha([r, 1))$. Suppose $\alpha$ can be chosen to satisfy the following conditions:

1. the point $\alpha(0)$ lies on the bottom of $X$; and

2. for all compact subsets $K$ of $X$, there exists $r \in[0,1)$ such that $F(r) \cap K=\emptyset$.

Then we say that $f$ is a fat flow. In essence, a fat flow has an underlying topological path whose image under the homeomorphism $\psi$ begins at $\left\{(x, y) \mid x^{2}+y^{2}=1\right\}$ and approaches $\left\{(x, y) \mid x^{2}+y^{2}=2\right\}$. If $w$ is a weight function on $X$, then the "fat $w$-height" $H_{w, f}$ of $X$ under $w$ is the infimum of $\operatorname{len}_{w} f$, where $f$ varies over all possible fat flows. Now define the fat flow modulus

$$
M_{f}=\sup _{w} \frac{H_{w, f}^{2}}{A_{w}}
$$

where the supremum is taken over all weight functions $w$ on $X$. If a weight function actually achieves this supremum, we call it a fat flow optimal weight function. One can see that the fat flow modulus is a combinatorial version of the classical modulus, which seeks to maximize the ratio of height squared to area.

The fat flow modulus was originally defined for tilings (or, in fact, shinglings) of topological rectangles and annuli (see [4]). In these cases we designate two opposite sides of the rectangle (or the two boundary components of the annulus) to be the top and bottom of the tiling; fat flows have underlying topological paths joining the top to the bottom. The height $H_{w, f}$ is the length of a minimal fat flow, and $M_{f}=\sup _{w}\left(H_{w, f}^{2} / A_{w}\right)$ as before. Cannon et al. show (in [4]) that fat flow optimal weight functions exist for all locally finite tilings of (closed) topological rectangles and annuli.

In this article we shall see that whether or not a half-open annular tiling has finite fat flow modulus depends on the degrees of the vertices of the tiling as one moves outwards from the boundary. In essence, the modulus is finite if the degrees are large, since large degrees cause the number of tiles to grow quickly. Otherwise the modulus is infinite.

We employ the following three theorems in our proofs. The first is found in [4] and [8]; the second is found in [2]; the third in [2] and [8].

Theorem 1. Let A be a locally finite tiling of a topological rectangle, annulus, or halfopen annulus. Let $w$ be a weight function on $A$. Let $p_{1}, p_{2}, \ldots, p_{k}$ be w-minimal fat flows in A. Suppose that $w(t)=\sum_{i=1}^{k} a_{i} p_{i}(t)$, for all tiles $t$ in $A$, where $a_{1}, \ldots, a_{k}$ are positive real numbers, and where $p_{i}(t)=1$ if $t$ is in $p_{i}$, and $p_{i}(t)=0$ otherwise. Then $w$ is a fat flow optimal weight function on A.

This theorem states, in essence, that fat flow optimal weight functions are weighted sums of their minimal fat flows.

Theorem 2 (Bounded Overlap Theorem). Suppose that a half-open annulus $X$ has two locally finite tilings $T$ and $T^{\prime}$, such that no element of $T$ intersects more than $K$ elements of $T^{\prime}$ and such that no element of $T^{\prime}$ intersects more than $K$ elements of $T$. Let $M(X, T)$ be the fat flow modulus of $X$ with the tiling $T$, and let $M\left(X, T^{\prime}\right)$ be the fat flow modulus 
of $X$ with the tiling $T^{\prime}$. Then

$$
M(X, T) \leq K^{3} \cdot M\left(X, T^{\prime}\right) .
$$

Theorem 3 (Layer Theorem). Suppose a half-open annulus $X$ with a locally finite tiling is divided into a family $\left\{X_{j}\right\}_{j=1}^{\infty}$ of tiled closed annuli, such that any two members of the family are disjoint except possibly at their boundaries. Then the fat flow modulus of $X$ is greater than or equal to the sum of the fat flow moduli of the sets $X_{j}$.

\section{A Conjecture by He and Schramm}

Following He and Schramm in [6], we define a disk triangulation graph to be the 1skeleton of a triangulation of an open topological disk. If $G$ is a disk triangulation graph and $v$ is a vertex of $G$, consider the tiling $T$ dual to $G$. If $t_{v}$ is the interior of the tile corresponding to $v$, then let $T_{v}=T \backslash\left\{t_{v}\right\}$. Now $T_{v}$ is a half-open annulus. We say that $G$ is parabolic if the fat flow modulus of $T_{v}$ is infinite for some vertex $v$ in $G$; otherwise, we say that $G$ is hyperbolic.

In their article He and Schramm use the term "vertex extremal length" to refer to combinatorial modulus, since their formulation assigns weights to the vertices of $G$; they consider paths in the graph rather than fat paths in the triangulation. In this paper, however, we assign weights to the faces of the triangulation and calculate moduli as described above. To see that this difference in formulation does not affect the parabolicity or hyperbolicity of the graph, suppose that $G$ is a locally finite disk triangulation graph with bounded valence. Suppose we remove a vertex from $G$. Then the resulting graph $\bar{G}$ is the 1-skeleton of a locally finite triangulation $T$ of a half-open annulus. Let $M_{v}$ be the modulus of $\bar{G}$ in terms of the He/Schramm formulation (with weights concentrated at the vertices and paths given by edges); and let $M_{t}$ be the fat flow modulus of the triangulation $T$ (viewed as a tiling, with weights assigned to the faces). Let $D$ be the dual tiling of the graph $\bar{G}$. Now $M_{v}$ is the fat flow modulus of $D$. Since $G$ has bounded valence, the tiling $T$ spanned by $\bar{G}$ has bounded overlap with the tiling $D$, and thus, by the Bounded Overlap Theorem, either $M_{v}$ and $M_{t}$ are both infinite or they are both finite. Hence, whether or not we assign weights to the vertices or to the tiles does not affect the type (parabolic or hyperbolic) of the graph.

One can also define parabolicity and hyperbolicity in terms of circle packings, in terms of "edge extremal length" (in which weights are assigned to the edges), in terms of simple random walks, and in terms of electrical networks. He and Schramm show in [6] that all of these formulations are equivalent.

In the same paper He and Schramm proceed to discuss the relationship between the vertex valences of a disk triangulation graph and the type (parabolic or hyperbolic) of the graph. They obtain the following results:

Theorem 4. Let $G$ be the 1-skeleton of a disk triangulation, and suppose that at most finitely many vertices in $G$ have valence greater than 6 . Then $G$ is parabolic.

Theorem 5. Let $G$ be the 1-skeleton of a locally finite disk triangulation. Let $\operatorname{val}(v)$ 
denote the valence of the vertex $v$. Suppose that

$$
\sup _{W_{0}} \inf _{W \supseteq W_{0}}\left(\frac{1}{|W|} \sum_{v \in W} \operatorname{val}(v)\right)>6,
$$

where $W$ and $W_{0}$ are nonempty finite connected sets of vertices. Then $G$ is hyperbolic.

They note the wide gap between these two theorems and speculate on the possibility of obtaining type criteria in terms of the sequence $\left\{a_{n}\right\}$, where

$$
a_{n}=\sum_{v}(\operatorname{val}(v)-6),
$$

the sum being taken over all vertices within $n$ edges of a specified base vertex $v_{0}$. In particular, they speculate that boundedness of $\left\{a_{n}\right\}$ implies parabolicity of the corresponding disk triangulation graph.

It is this conjecture which we prove. We state it as the following theorem.

Theorem 6. Let $G$ be a disk triangulation graph of bounded valence. Let $v_{0}$ be a vertex of $G$. For any vertex $v$ of $G$, let $|v|$ denote the minimum number of edges in a path connecting $v_{0}$ to $v$. Let

$$
a_{n}=\sum_{|v| \leq n}(\operatorname{val}(v)-6) .
$$

If the sequence $\left\{a_{n}\right\}$ is bounded, then $G$ is parabolic.

We call $a_{n}$ the valence excess.

\section{Discussion of Theorem 6}

To understand more clearly what this theorem is saying, we consider the behavior of the sequence $\left\{a_{n}\right\}$. This sequence is not necessarily well-behaved, for it can be unbounded and yet have a constant subsequence. For an example of such a triangulation, see Fig. 1. Identify the sides to form a disk triangulation graph. If the vertex at the bottom is the base vertex, then the numbers at the side give the valence excess. Note that $\lim \sup a_{n}=\infty$, whereas $\lim \inf a_{n}=-6$. So, although $\left\{a_{n}\right\}$ is not bounded, it has a subsequence that is bounded.

Moreover, one can use the Bounded Overlap Theorem to see that the tiling has finite fat flow modulus and is therefore hyperbolic. To do so, consider the "squared rectangle" $R$ shown in Fig. 2. Note that $R$ has bounded overlap with the triangulation shown in Fig. 1. The weight function determined by the sizes of the squares in the figure is the fat flow optimal weight function on $R$. If the largest square in $R$ has unit side length, then the height of $R$ is 6 , and its area is 36 . The fat flow modulus is 1 , and therefore the original triangulation must be hyperbolic. Thus, even if $\left\{a_{n}\right\}$ has a bounded subsequence we are not guaranteed parabolicity.

However, consider $R$ more closely. Notice that it consists of concentric layers (which become annuli after we identify the sides); the sum of the heights of these annuli gives the height of the entire squared rectangle. Furthermore, notice that the valence excess 


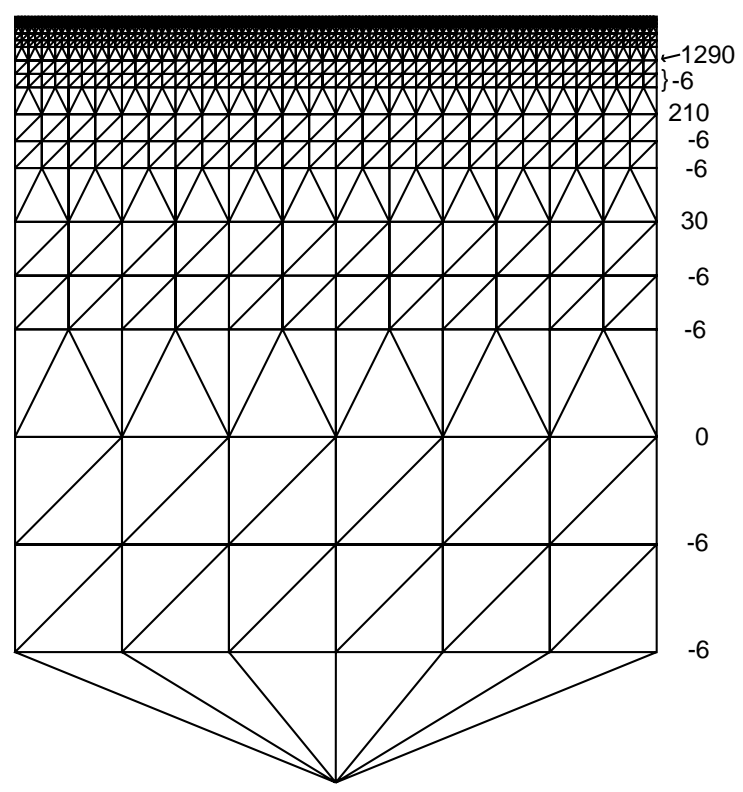

Fig. 1. A hyperbolic disk triangulation graph.

increases only when the number of tiles in a layer increases. When the number of tiles in a layer remains the same, the valence excess stays at -6 .

Suppose that we modify $R$. Instead of doubling the number of tiles every third layer, let the number of layers between each doubling be described by a sequence $r_{n}$. In particular, we say that the number of layers with $6 \cdot 2^{n-1}$ tiles is $r_{n}$. Now, if we have a layer in our modified rectangle with $6 \cdot 2^{n-1}$ tiles, the height of that layer is $2^{-n+1}$. So the height of

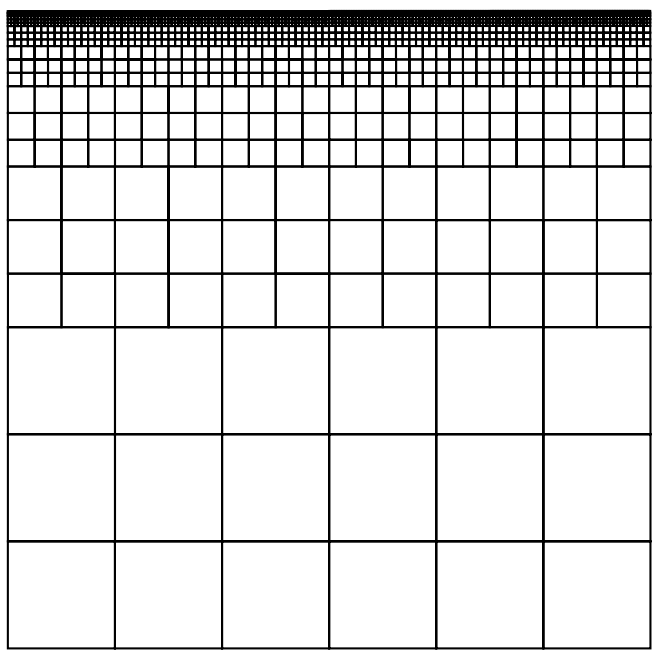

Fig. 2. A squared rectangle $R$ corresponding to Fig. 1 . 
the total modified rectangle is

$$
\sum_{n=1}^{\infty} r_{n} 2^{-n+1} .
$$

If $r_{n}$ increases as a polynomial, for instance, the height of the rectangle (and hence its modulus) will be finite. Recall that the only times $\left\{a_{n}\right\}$ was greater than -6 was when we doubled a layer; if $r_{n}$ grows like a polynomial, then, we will have arbitrarily long strings of values of -6 in $\left\{a_{n}\right\}$; nevertheless, the tiling will still be hyperbolic.

Looking at these examples gives us an idea of how to approach the proof of Theorem 6. We decompose the disk triangulation graph (and the tiling spanned by it) into concentric annuli and then estimate the fat flow modulus of each of these annuli. The Layer Theorem allows us to sum their moduli, obtaining an estimate for the modulus of the entire tiling. To estimate the moduli of the annuli, note that in the above example, the number of tiles in the annulus controlled the modulus (height); at the same time, the number of vertices on the borders of the annuli controlled the valence excess. Thus, if we assume the valence excess to be bounded, we can estimate the number of vertices; these estimates, in turn, give us information about the disposition of the tiles in each annulus, allowing us to estimate moduli. It is this general plan of attack which we follow below.

\section{Triangulated Annuli}

Let $A$ be a closed topological annulus with two (disjoint) boundary components homeomorphic to circles. Construct a homeomorphism from $A$ to a simplicial 2-complex, thus triangulating $A$. Then we say that $A$, with this triangulation, is a simply triangulated annulus if all vertices of the triangulation lie on the boundary of $A$ and if any edges joining a top vertex to another top vertex are themselves part of the top.

We consider the types of triangles that a simply triangulated annulus can contain. First, we know that at least one vertex of each triangle must lie on the bottom of $A$ by definition. Now if we suppose that exactly one vertex lies on the bottom, then two vertices must lie on the top. The edges connecting those two top vertices must be part of the top boundary component. We call such a triangle a down triangle. (See Fig. 3.)

On the other hand, if a triangle has exactly two vertices on the bottom and, consequently, one vertex on the top, then we have two possible cases: either the edge connecting the two bottom vertices lies in the bottom boundary component, or it does not. We call the first sort of triangle an up triangle; we call the other sort a mid triangle. Finally, if all three vertices lie on the bottom, then we call the triangle a bent triangle. (See Fig. 4.)
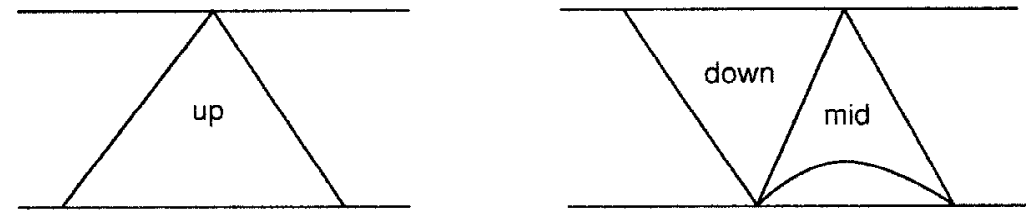

Fig. 3. Up, down, and mid triangles. 

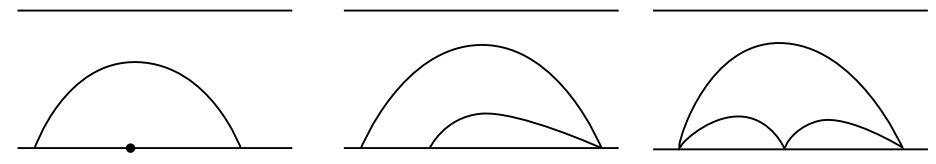

Fig. 4. Bent triangles.

These are the only types of triangles that can occur in a simply triangulated annulus. Incidentally, this analysis shows us that all vertices in a simply triangulated annulus are either on the bottom or are connected to the bottom by an edge.

We define a well-triangulated annulus to be a closed topological annulus with two (disjoint) boundary components and an imposed triangulation such that all vertices that do not lie on the bottom are connected to the bottom by an edge; and such that any edges joining a top vertex to another top vertex are themselves part of the top. For each vertex $v$ of a well-triangulated annulus, we define the tile valence of $v$ (denoted tval $(v)$ ) to be the number of tiles (triangles) in the annulus incident to $v$. Note that all simply triangulated annuli are well-triangulated.

We begin by considering simply triangulated annuli such that all nonboundary edges connect the bottom and the top of the annulus.

Proposition 7. Let $A$ be a simply triangulated annulus such that all nonboundary edges of the triangulation connect the bottom and the top of the annulus. Suppose the bottom of the annulus contains $k$ vertices, labeled $b_{1}, b_{2}, \ldots, b_{k}$, and suppose the top contains $n$ vertices, labeled $t_{1}, t_{2}, \ldots, t_{n}$. Then

$$
\sum_{i=1}^{k}\left(\operatorname{tval}\left(b_{i}\right)-3\right)=n-k
$$

and

$$
\sum_{i=1}^{n}\left(\operatorname{tval}\left(t_{i}\right)-3\right)=k-n
$$

Proof. Choose an orientation for the annulus and order the nonboundary edges clockwise. Re-indexing if necessary, we assume that an edge connects $b_{1}$ and $t_{1}$, that the other vertices $b_{2}, \ldots, b_{k}$ and $t_{2}, \ldots, t_{n}$ are ordered consecutively clockwise around the annulus, and that no edge connects $b_{1}$ to $t_{n}$. (That is, the edge from $b_{1}$ to $t_{1}$ is the "first" edge clockwise from $b_{1}$.) Now for $i=1, \ldots, k$, we let $m_{i}$ be the number of nonboundary edges incident to $b_{i}$. Note that $\operatorname{tval}\left(b_{i}\right)=m_{i}+1$. (See Fig. 5.)

Now for $i=1$, the last edge from $b_{1}$ hits $t_{m_{1}}$, as does the first edge from $b_{2}$. By induction one can show that the last edge of $b_{i}$ hits $t_{m_{1}+\cdots+m_{i}-(i-1)}$ for $i=1, \ldots, k$. However, the last edge from $b_{k}$ must hit $t_{1}=t_{n+1}$. So

$$
\begin{aligned}
\sum_{i=1}^{k} m_{i}-(k-1) & =n+1, \\
\sum_{i=1}^{k} m_{i}-k & =n .
\end{aligned}
$$




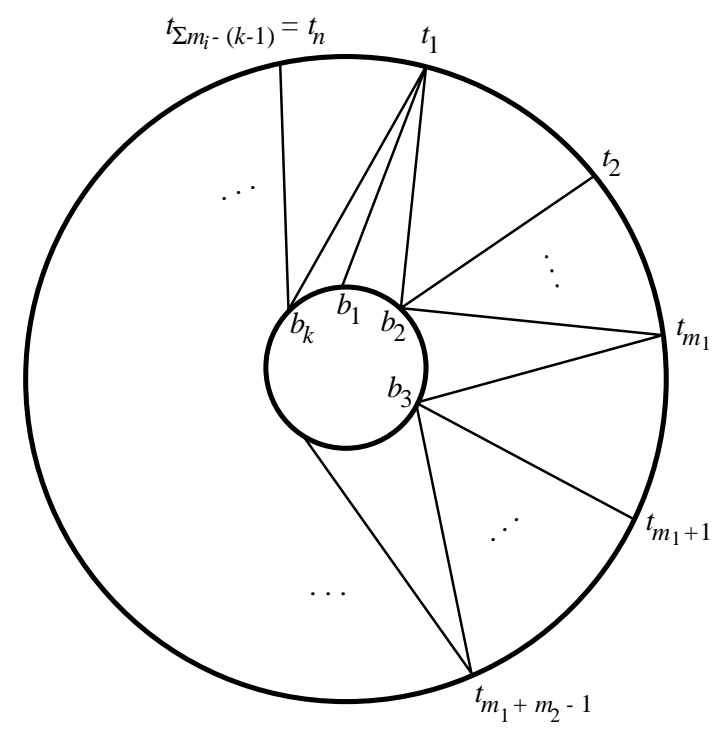

Fig. 5. Arrangement of edges and vertices on $A$.

Since $\operatorname{tval}\left(b_{i}\right)=m_{i}+1$,

$$
\begin{aligned}
\sum_{i=1}^{k}\left(\operatorname{tval}\left(b_{i}\right)-3\right) & =\sum_{i=1}^{k}\left(m_{i}+1-3\right) \\
& =\sum_{i=1}^{k} m_{i}-2 k=n-k .
\end{aligned}
$$

The other half of the proposition follows by symmetry.

Our next task is to extend this proposition to deal with any simply triangulated annulus. To do so involves some valence count calculations. Since this type of argument recurs throughout this paper, we refer to following standard lemma:

Lemma 8 (Shelling Lemma for Triangulated 2-Disks). Suppose that $D$ is a disk with triangulation $T$. If $T$ comprises more than one triangle, then there is a triangle $\Delta$ in $T$ with the following property: the intersection of $\partial \Delta$ with the interior of $D$ is an open arc consisting of either the interior of one edge of $\Delta$ (type one) or the interior of two edges and the intervening vertex (type two). In fact, there are two such triangles $\Delta$.

These triangles $\Delta$ are called shelling disks. One can prove this lemma by induction on the number of triangles in the triangulation $T$. The fact that there are at least two shelling disks is the key to the induction. The actual proof is left to the reader.

One can extend the concept of a shelling disk to triangulated annuli as follows. In a triangulated annulus $A$, a shelling disk is a triangle $\Delta$ with the following properties. 
First, the intersection of $\Delta$ with $\partial A$ is an arc in the bottom of $A$. Second, the intersection of $\partial \Delta$ with int $A$ is an open arc which consists of either the interior of one edge of $\Delta$ (type one) or the interior of two edges and the intervening vertex (type two). Note that in the case of triangulated annuli, we are not guaranteed the existence of shelling disks.

We use the following procedure at several points in the paper. For convenience, we label it the Uniform Reduction Procedure:

Uniform Reduction Procedure. If a shelling disk $\Delta$ is of type one, we simplify the disk or annulus by removing $\Delta$ and then taking the closure of its complement. If the shelling disk $\Delta$ has type two, we simplify the disk or annulus by collapsing it to an arc which starts at the boundary and ends at an interior vertex; to do so, we identify the two interior edges of the shelling disk and collapse the boundary edge to a single point.

Our first use of the shelling lemma and the uniform reduction procedure is in extending Proposition 7 to all simply triangulated annuli.

Proposition 9. Let $A$ be a simply triangulated annulus. Suppose the bottom of the annulus contains $k$ vertices, labeled $b_{1}, b_{2}, \ldots, b_{k}$, and suppose the top contains $n$ vertices, labeled $t_{1}, t_{2}, \ldots, t_{n}$. Let $q$ be the number of bottom vertices connected to the top by an edge. Then

$$
\sum_{i=1}^{k}\left(\operatorname{tval}\left(b_{i}\right)-3\right)=n-q
$$

and

$$
\sum_{i=1}^{n}\left(\operatorname{tval}\left(t_{i}\right)-3\right)=q-n
$$

Proof. We perform induction on the number of interior edges connecting the bottom to the bottom. If every interior edge connects the bottom to the top, then Proposition 7 applies. Now assume that we have proved our proposition for all simply triangulated annuli with at most $n-1$ interior edges connecting the bottom to the bottom $(n \geq 1)$. Suppose $A$ has $n$ interior edges connecting bottom to bottom. Consider one of these interior edges. It must bound a triangulated disk $D$ in $A$. Either $D$ is already a shelling disk for $A$ or, by the shelling lemma, $D$ contains two shelling disks for $D$, one of which, $\Delta$, must be a shelling disk for $A$. Since there are no interior vertices in $A, \Delta$ must be of type one. Delete $\Delta$ from $A$ as specified in the uniform reduction procedure to obtain $A^{\prime}$. By removing the type one shelling disk $\Delta$, we have reduced the number of interior edges connecting bottom to bottom by one, and the proposition is true of $A^{\prime}$ by our induction. A simple calculation shows that the sums which appear in the conclusion of the proposition do not change in passing from $A$ to $A^{\prime}$.

To conclude this section, we consider well-triangulated annuli, thus allowing for interior vertices. 

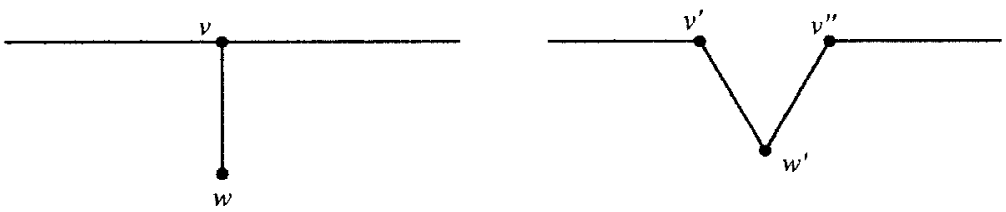

Fig. 6. Removing an interior vertex.

Proposition 10. Let A be a well-triangulated annulus. Suppose this triangulation contains $n$ top vertices, $c$ bottom vertices, and $x$ interior vertices. Label the bottom vertices $v_{1}, \ldots, v_{c}$. Then

$$
\sum_{i=1}^{c}\left(\operatorname{tval}\left(v_{i}\right)-3\right) \geq n+x-c .
$$

Proof. If $A$ is a simply triangulated annulus, then the result follows from Proposition 9. So we may perform induction on the number of interior vertices. Suppose we have proved the proposition for all well-triangulated annuli with $n$ interior vertices. Now suppose $A$ has $n+1$ interior vertices.

We first consider the possibility that one of the interior vertices is connected by an edge to a top vertex. Let $w$ be such an interior vertex of $A$, connected to a top vertex $v$ by an edge. Replace $v$ by two top vertices $v^{\prime}$ and $v^{\prime \prime}$, and replace $w$ by a top vertex $w^{\prime}$ connected to both $v^{\prime}$ and $v^{\prime \prime}$ (see Fig. 6), thus transforming an interior vertex into two top vertices without changing the $\operatorname{sum} \sum_{i=1}^{c}\left(\operatorname{tval}\left(v_{i}\right)-3\right)$. By induction, the resulting annulus (which has one fewer interior vertices) satisfies the conclusion of the proposition. Since none of the bottom vertices has changed, we know that $A$ must therefore also satisfy the conclusion.

Now suppose none of the $n+1$ interior vertices of $A$ is joined by an edge to a top vertex. In this case let $B$ be the union of all triangles which intersect the top. $B$ is an annulus with the following property: if $D$ is the closure of a component of $A \backslash B$, then $D$ is a triangulated disk with exactly one edge on $B$ and the complementary portion of the boundary on the bottom of $A$.

Let $k$ be the greatest number of triangles in any of these disks $D$. We perform induction on $k$. Since $A$ contains interior vertices, none of which is connected to the top by an edge, we conclude that $k$ must be at least two. If $k=2$, then each $D$ can contain at most one interior vertex, but such disks $D$ add 4 to the sum on the left-hand side of the proposition. Thus the proposition would be true. Now suppose that we have shown the proposition true for some $k \geq 2$. If there exists a $D$ with $k+1$ triangles, then apply the shelling lemma to $D$ in order to supply a shelling disk. Now we may simplify $D$ (and hence $A$ ) by the uniform reduction procedure. By our induction on the number of triangles in $D$, the result is true for the simplified annulus. Two straightforward calculations (one for each type of shelling disk) show that the result is therefore true for $A$. Hence we have now finished our induction on the number of interior vertices, establishing the truth of the proposition for all well-triangulated annuli. 

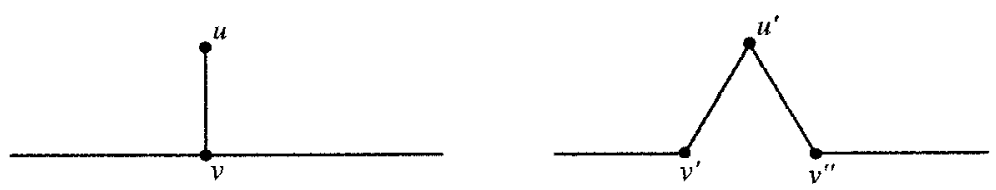

Fig. 7. Removing an interior vertex.

Finally, we must establish one more result about well-triangulated annuli.

Proposition 11. Let A be a well-triangulated annulus. Suppose A contains c bottom vertices, labeled $v_{1}, \ldots, v_{c} ; n$ top vertices, labeled $w_{1}, \ldots, w_{n}$; and $x$ interior vertices, labeled $u_{1}, \ldots, u_{x}$. Then

$$
\sum_{i=1}^{c}\left(\operatorname{tval}\left(v_{i}\right)-3\right)+\sum_{i=1}^{x}\left(\operatorname{tval}\left(u_{i}\right)-6\right)=-\sum_{i=1}^{n}\left(\operatorname{tval}\left(w_{i}\right)-3\right) .
$$

Proof. Let $u$ be an interior vertex of $A$. Since $A$ is a well-triangulated annulus, there must be an edge connecting $u$ to a bottom vertex $v$. We remove the interior vertex $u$ somewhat as before, replacing $v$ by two bottom vertices $v^{\prime}$ and $v^{\prime \prime}$, and replacing $u$ by a bottom vertex $u^{\prime}$ connected to $v^{\prime}$ and $v^{\prime \prime}$. (See Fig. 7.)

Notice that $\operatorname{tval}(v)=\operatorname{tval}\left(v^{\prime}\right)+\operatorname{tval}\left(v^{\prime \prime}\right)$ and $\operatorname{tval}(u)=\operatorname{tval}\left(u^{\prime}\right)$. Therefore we can see that $(\operatorname{tval}(v)-3)+(\operatorname{tval}(u)-6)=\left(\operatorname{tval}\left(v^{\prime}\right)-3\right)+\left(\operatorname{tval}\left(v^{\prime \prime}\right)-3\right)+\left(\operatorname{tval}\left(u^{\prime}\right)-3\right)$. Hence, this slicing operation has not affected the sum $\sum\left(\operatorname{tval}\left(v_{i}\right)-3\right)+\sum\left(\operatorname{tval}\left(u_{i}\right)-6\right)$; nor has it changed the sum $\sum\left(\operatorname{tval}\left(w_{i}\right)-3\right)$, since it did not touch the top of the annulus.

Performing this slicing operation for all interior vertices, we obtain a new annulus $A^{\prime}$ with no interior vertices. It has the same $n$ top vertices $w_{1}, \ldots, w_{n}$; and it has $c+2 x$ bottom vertices, which we label $v_{1}^{\prime}, \ldots, v_{c+2 x}^{\prime}$. Since $A^{\prime}$ is a simply triangulated annulus, Proposition 9 applies. Let $q$ be the number of bottom vertices (in $A^{\prime}$ ) which are connected to the top. We see that

$$
\sum_{i=1}^{c}\left(\operatorname{tval}\left(v_{i}\right)-3\right)+\sum_{i=1}^{x}\left(\operatorname{tval}\left(u_{i}\right)-6\right)=\sum_{i=1}^{c+2 x}\left(\operatorname{tval}\left(v_{i}^{\prime}\right)-3\right)=n-q .
$$

However, again by Proposition 9, we know that $\sum_{i=1}^{n}\left(\operatorname{tval}\left(w_{i}\right)-3\right)=q-n$, and hence we conclude that

$$
\sum_{i=1}^{c}\left(\operatorname{tval}\left(v_{i}\right)-3\right)+\sum_{i=1}^{x}\left(\operatorname{tval}\left(u_{i}\right)-6\right)=-\sum_{i=1}^{n}\left(\operatorname{tval}\left(w_{i}\right)-3\right) .
$$

\section{The Moduli of Well-Triangulated Annuli}

In this section we obtain a lower bound on the modulus of any well-triangulated annulus in terms of the number of vertices on the top and bottom. First we consider simply triangulated annuli. 
We call a sequence of alternating up and down triangles a basic subtiling of a simply triangulated annulus. (We require a basic subtiling to include at least one up triangle and at least one down triangle.) If the entire annulus is a basic subtiling, we give it the same top and bottom as the original annulus and proceed to compute its fat flow modulus. Otherwise, we consider the basic subtiling to be a quadrilateral, with top and bottom inherited from the annulus. Our first proposition deals with an optimal weight function for basic subtilings:

Proposition 12. Consider a basic subtiling containing $m>0$ down triangles and $n>0$ up triangles. Then an optimal weight function for this basic subtiling is

$$
w(t)= \begin{cases}n & \text { ift is a down triangle, } \\ m & \text { ift is an up triangle. }\end{cases}
$$

Proof. There are four possible cases:

1. The basic subtiling is a quadrilateral with $m=n+1$.

2. The basic subtiling is a quadrilateral with $m=n-1$.

3. The basic subtiling is a quadrilateral with $m=n$.

4. The basic subtiling is an annulus.

For each case we can show that the function $w(t)$ defined above is an optimal weight function (according to Theorem 1) by expressing it as a sum of its minimal fat flows. Note that by the "fatness" of these paths, all minimal fat flows must contain both an up and a down triangle. In particular, observe that minimal fat flows (from bottom to top) in a basic subtiling begin at an up triangle and end at a down triangle.

Our first case, illustrated by Fig. 8 , is a basic subtiling which is a quadrilateral with $m=n+1$. Note that each of the flows shown in the figure is a $w$-minimal fat flow. Consider one of the down triangles, which we call $t$. We find that

$$
\begin{aligned}
\sum_{i=1}^{n}(n-i & +1) p_{2 i-1}(t)+\sum_{i=1}^{n} i p_{2 i}(t) \\
& = \begin{cases}n-1+1 & \text { if } t \text { is the leftmost down triangle, } \\
n & \text { if } t \text { is the rightmost down triangle, } \\
i+(n-(i+1)+1) & \text { otherwise }\end{cases} \\
=n=w(t) . &
\end{aligned}
$$

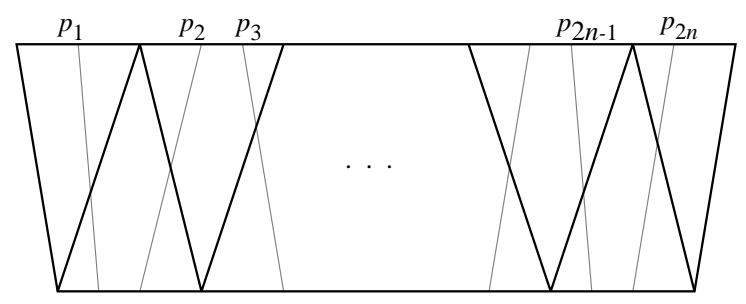

Fig. 8. Basic subtiling with $m=n+1$. 
On the other hand, if $t$ is one of the up triangles in this basic subtiling, then

$$
\begin{aligned}
\sum_{i=1}^{n}(n-i+1) p_{2 i-1}(t)+\sum_{i=1}^{n} i p_{2 i}(t) & =(n-i+1)+i \\
& =n+1=m=w(t)
\end{aligned}
$$

In either case, $w=\sum_{i=1}^{n}(n-i+1) p_{2 i-1}+\sum_{i=1}^{n} i p_{2 i}$; thus $w$ is the weighted sum of $w$-minimal flows, and it is therefore an optimal weight function for this basic subtiling.

The other three cases-when $m=n-1$, when $m=n$ in a quadrilateral, and when the basic subtiling is an annulus_-are handled similarly.

Corollary 13. If $B$ is a basic subtiling consisting of $m>0$ down triangles and $n>0$ up triangles, then the modulus $M$ of $B$ is

$$
\frac{n+m}{n m} \text {. }
$$

Proof. By Proposition 12 the optimal weight function $w$ on $B$ assigns a weight of $n$ to the down triangles and a weight of $m$ to the up triangles. Since a fat flow will contain one up triangle and one down triangle, the length of any fat flow is $n+m$, and $H_{w, f}(B)=n+m$. The area $A_{w}(B)$ will be $m n^{2}+n m^{2}$. The result follows.

Now partially order the basic subtilings by subset inclusion and obtain maximal basic subtilings, which would have no tiles in common. Suppose that no nonboundary edges of a simply triangulated annulus join the bottom to the bottom; then the triangulation contains only up triangles and down triangles.

Proposition 14. Let $A$ be a simply triangulated annulus such that all nonboundary edges of the triangulation connect the bottom and the top of the annulus. Let the maximal basic subtilings of $A$ be denoted $B_{1}, B_{2}, \ldots, B_{k}$. For $i=1, \ldots, k$, let $n_{i}$ and $m_{i}$ be the number of up and down triangles, respectively, in $B_{i}$. Then an optimal weight function $w(t)$ for $A$ is given as follows:

$$
w(t)= \begin{cases}\frac{m_{i}}{n_{i}+m_{i}} & \text { if } t \text { is an up triangle in } B_{i} \\ \frac{n_{i}}{n_{i}+m_{i}} & \text { if } t \text { is a down triangle in } B_{i} \\ 0 & \text { otherwise. }\end{cases}
$$

Proof. Observe that $w$ is well-defined since maximal basic subtilings cannot share tiles. Note further that $w$ is simply the concatenation of the optimal weight functions on the maximal basic subtilings, all normalized to have a height of one. Thus, in order to prove the proposition, we need to show that one cannot construct a path with $w$-length less than one by starting in one maximal basic subtiling and ending in another.

Suppose we have a path $p$ which is not contained in a maximal basic subtiling. It must begin with an up triangle $t$. First suppose that $t$ is part of a maximal basic subtiling. Then 


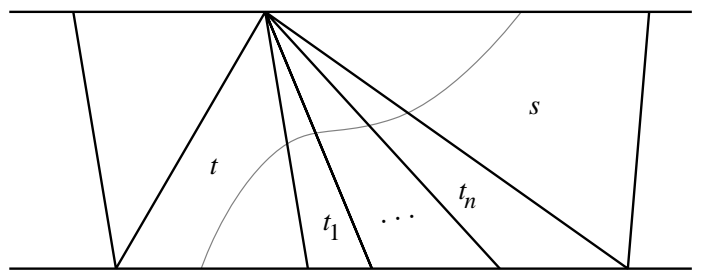

Fig. 9. A flow out of a maximal basic subtiling.

$t$ must be at the edge of that maximal basic subtiling; otherwise the second tile in $p$ would be a down triangle in the same maximal basic subtiling, and the path's length would be one. Furthermore, the path must end at the first down triangle $s$ that it encounters, since by then it shall have reached the top of the annulus. Thus, the path must look like the one shown in Fig. 9. The path consists of triangles $\left\{t, t_{1}, \ldots, t_{n}, s\right\}$. However, the last two tiles $\left(t_{n}\right.$ and $\left.s\right)$ of $p$ are an alternating up/down pair of triangles; thus they are themselves part of a maximal basic subtiling. Hence $w\left(t_{n}\right)+w(s)$ must equal one, and the length of $p$ therefore cannot be less than one.

One can handle the other case (in which the first triangle of $p$ is not part of a maximal basic subtiling) similarly. Therefore, we may conclude that paths of length one are $w$ minimal, and hence that the function $w$, being the sum of $w$-minimal fat flows, is an optimal weight function for $A$.

Corollary 15. Let A be a simply triangulated annulus such that all nonboundary edges of the triangulation connect the bottom and the top of the annulus. Suppose A contains $k$ maximal basic subtilings, and let $M_{i}$ be the fat flow modulus of the $i$ th maximal basic subtiling, for $i=1, \ldots, k$. Then the fat flow modulus of $A$ is

$$
\left(\sum_{i=1}^{k} \frac{1}{M_{i}}\right)^{-1}
$$

Proof. Proposition 14 states that the optimal weight function $w$ for $A$ is the concatenation of the optimal weight functions for the maximal basic subtilings, each one normalized to unit height. Note that the height of the $i$ th maximal basic subtiling under $w$ will be 1 , and so its area will be $1 / M_{i}$. The height of $A$ under $w$ will also be 1 . The area of $A$ will be the sum of the areas of the maximal basic subtilings, namely, $\sum 1 / M_{i}$, and the modulus of $A$ will be the reciprocal of that sum.

Now that we have an expression for the modulus of $A$, we consider the minimum possible modulus for such a simply triangulated annulus, in terms of the number of vertices on the top and the number of vertices on the bottom.

Proposition 16. Let A be a simply triangulated annulus such that all nonboundary edges of the triangulation connect the bottom and the top of the annulus. Suppose A has $n$ vertices on the top and $k$ vertices on the bottom. If $M$ is the fat flow modulus of $A$, then $M \geq 3 /(2 m)$, where $m=\min \{n, k\}$. 
Proof. Note that $n$ and $k$ also denote the number of down and up triangles, respectively, in $A$. Denote the maximal basic subtilings of $A$ as $B_{1}, B_{2}, \ldots, B_{p}$. For $i=1, \ldots, p$, let $n_{i}$ and $k_{i}$ be the number of up and down triangles, respectively, in $B_{i}$. By Corollary 13 the fat flow modulus for $B_{i}$ is

$$
M_{i}=\frac{n_{i}+k_{i}}{n_{i} k_{i}} .
$$

Let $m_{i}=\min \left\{n_{i}, k_{i}\right\}$. We have three possible cases: either $m_{i}=n_{i}=k_{i} ; m_{i}=n_{i}=$ $k_{i}-1$; or $m_{i}=k_{i}=n_{i}-1$. Consider the first case. If $m_{i}=n_{i}=k_{i}$, then $M_{i}=2 / m_{i}$. The second and third cases reduce to the same situation:

$$
M_{i}=\frac{2 m_{i}+1}{m_{i}\left(m_{i}+1\right)}<\frac{2}{m_{i}} .
$$

We conclude that

$$
\frac{2 m_{i}+1}{m_{i}\left(m_{i}+1\right)} \leq M_{i}
$$

Now since $m_{i} \geq 1$, we know

$$
\begin{aligned}
2 m_{i}\left(2 m_{i}+1\right) & \geq 3\left(m_{i}^{2}+m_{i}\right), \\
M_{i} \geq \frac{2 m_{i}+1}{m_{i}^{2}+m_{i}} & \geq \frac{3}{2 m_{i}}, \\
\frac{1}{M_{i}} & \leq \frac{2 m_{i}}{3},
\end{aligned}
$$

for any $i$ from 1 to $p$. It therefore follows that

$$
\sum_{i=1}^{p} \frac{1}{M_{i}} \leq \sum_{i=1}^{p} \frac{2 m_{i}}{3} \leq \frac{2}{3} m
$$

since $m$, the minimum of the total number of up triangles and the total number of down triangles in $A$, must be at least as large as the sum of the minima for each maximal basic subtilings (i.e., at least as large as $\sum m_{i}$ ).

However, by Corollary 15, we have

$$
M=\left(\sum_{i=1}^{p} \frac{1}{M_{i}}\right)^{-1} \geq\left(\frac{2}{3} m\right)^{-1}=\frac{3}{2 m},
$$

which is what was to be proven.

Note that this estimate is the best possible; one can obtain a simply triangulated annulus with this modulus by taking each $n_{i}$ to be equal to 1 and letting each $k_{i}=2$. Then $m_{i}=1$ for all $i=1, \ldots, m, M_{i}=\frac{3}{2}$, and $\sum m_{i}=m$, making all the inequalities into equations.

The next proposition investigates the effect of ignoring bent triangles and considering mid triangles as up triangles. 
Proposition 17. Let $X$ be a simply triangulated annulus. Let $\bar{X}$ be the simply triangulated annulus obtained by deleting all bent triangles from $X$. Let $M$ and $\bar{M}$ be the fat flow moduli of $X$ and $\bar{X}$, respectively. Then $M \geq \bar{M}$.

Proof. Let $\bar{w}$ be a fat flow optimal weight function for $\bar{X}$. Then $\bar{M}$ is the ratio of $H_{\bar{w}, f}^{2}(\bar{X})$ to $A_{\bar{w}}(\bar{X})$. Now define a weight function $w$ on $X$ by

$$
w(t)= \begin{cases}\bar{w}(t), & t \in \bar{X} \\ 0, & t \notin \bar{X}\end{cases}
$$

Clearly $A_{\bar{w}}(\bar{X})=A_{w}(X)$. Let $f$ be a $w$-minimal fat flow in $X$; then the restriction of $f$ to $\bar{X}$ is a $\bar{w}$-minimal fat flow in $\bar{X}$. Therefore $H_{w}(X)=H_{\bar{w}}(\bar{X})$. Now we may conclude,

$$
M=\sup _{v} \frac{H_{v}^{2}(X)}{A_{v}(X)} \geq \frac{H_{w}^{2}(X)}{A_{w}(X)}=\frac{H_{\bar{w}}^{2}(\bar{X})}{A_{\bar{w}}(\bar{X})}=\bar{M} .
$$

We may now estimate the modulus of any simply triangulated annulus.

Corollary 18. Let $X$ be a simply triangulated annulus with $n$ top vertices and with $q$ of the bottom vertices connected to the top. If $m$ is the minimum of $n$ and $q$, and if $M$ is the fat flow modulus of $X$, then $M \geq 3 /(2 m)$.

Proof. Define $\bar{X}$ and $\bar{M}$ as in Proposition 17. Then $\bar{X}$ is a simply triangulated annulus, all of whose nonboundary edges connect the bottom to the top. It has $n$ top vertices and $q$ bottom vertices. By Propositions 16 and 17 we have $M \geq \bar{M} \geq 3 /(2 m)$.

Finally, we obtain a result on the moduli of well-triangulated annuli.

Proposition 19. Let $X$ be a well-triangulated annulus. Suppose the triangulation contains $n$ top vertices. Let $M$ be the fat flow modulus of $X$. Then $M \geq 3 /(2 n)$.

Proof. Let $q$ be the number of bottom vertices which are connected to the top by an edge. As in the proof of Proposition 11, replace $X$ by a simply triangulated annulus $X^{\prime}$, with $n$ top vertices and $q+r$ bottom vertices connected to the top. Now if $m$ is the minimum of $n$ and $q+r$, then clearly $m \leq n$. By Corollary 18 the fat flow modulus $M^{\prime}$ of $X^{\prime}$ is greater than or equal to $3 /(2 m)$.

Let $w$ be the optimal weight function for $X^{\prime}$. Because of the procedure we used in replacing $X$ by $X^{\prime}$, there is a one-to-one correspondence of tiles in $X$ onto tiles in $X^{\prime}$. So we may apply the function $w$ to the original tiling $X$ as well as to the modified tiling $X^{\prime}$ and say that the $w$-areas of the two tilings are the same: $A_{w}(X)=A_{w}\left(X^{\prime}\right)$.

Next consider the relationship of flows in $X$ to flows in $X^{\prime}$. The top tiles of each tiling are the same, and all bottom tiles of $X$ are bottom tiles of $X^{\prime}$. The operation preserves all combinatorics, except that two tiles adjacent along some interior edge in $X$ may no longer be adjacent in $X^{\prime}$. However, both of these tiles will now be bottom tiles, so any 
fat flow in $X$ containing one of these tiles has a subset that is a fat flow in $X^{\prime}$. Thus, any fat flow in $X$ contains a fat flow in $X^{\prime}$. So the infimum of the $w$-lengths of the fat flows may be smaller when taken over $X^{\prime}$ than when taken over $X$; hence we conclude that $H_{w}(X) \geq H_{w}\left(X^{\prime}\right)$. Thus,

$$
M \geq \frac{H_{w}^{2}(X)}{A_{w}(X)} \geq \frac{H_{w}^{2}\left(X^{\prime}\right)}{A_{w}(X)}=M^{\prime} \geq \frac{3}{2 m} \geq \frac{3}{2 n} .
$$

At this point, it is possible to prove Theorem 6 if we assume one additional hypothesis-namely, that one can break the disk triangulation graph into concentric welltriangulated annuli surrounding the base vertex $v_{0}$ (see [8] for the proof). In the next section we work to remove this extra hypothesis. To do so, we need to consider the structure of a disk triangulation graph in detail.

\section{Dealing with Islands}

We define a well-triangulated annulus with islands to be a closed topological annulus with two (disjoint) boundary components homeomorphic to circles and an imposed triangulation which satisfies the following conditions: any edge joining a vertex on the top to another vertex on the top must be part of the top; and any vertex not connected to the bottom by an edge is either part of the bottom or surrounded by a cycle of vertices satisfying the following two conditions:

1. The vertices in this cycle contain no bottom vertices.

2. The vertices in this cycle are all connected to the bottom by an edge.

If $L$ is the union of such a cycle with its interior, then we say $L$ is an island. (See Fig. 10.) Note that by this definition all islands must contain vertices in their interior and be simply connected. The size of an island is the number of vertices in its boundary cycle.

If we take a disk triangulation graph $G$ and pick a base vertex $v_{0}$, then for any other vertex $v$, we may define $|v|$ to be the edge distance (minimum number of edges) from $v_{0}$ to $v$. Then we may define sets of vertices $V_{i}=\{v|| v \mid=i\}$. Suppose that $A$, a subset of the complex spanned by $G$, is a well-triangulated annulus with islands. Suppose that all vertices on the bottom of $A$ are in $V_{k}$ and that the other vertices in $A$ are in $\bigcup_{j=k+1}^{\infty} V_{j}$.

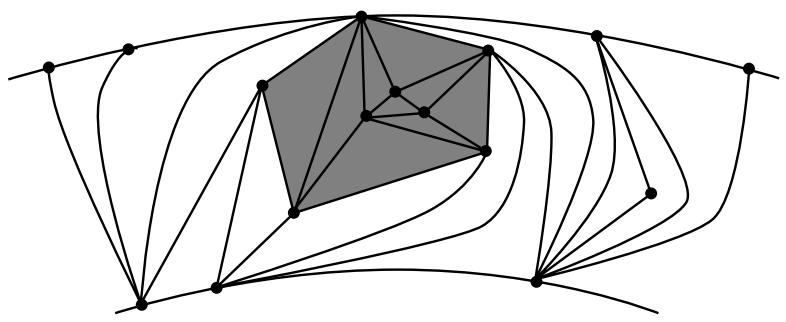

Fig. 10. An island 
If $L$ is an island in $A$, then we say that $L$ has station $k$ or is stationed at $k$. Note that all boundary vertices of such an island will belong to $V_{k+1}$. Suppose that $l$ is the maximum value such that there is a vertex in $L$ belonging to $V_{l}$. Then we say that the depth of $L$ is $l-(k+1)$. Note that all vertices in the interior of $L$ are in $\bigcup_{j=k+2}^{l} V_{k}$.

We now show that one can decompose $G$ into the union of a disk and a series of well-triangulated annuli with islands. In order to do so, we first present the following lemmas, whose proofs are left to the reader.

Lemma 20 (Annulus Construction Lemma). Suppose $X$ is a half-open annulus whose boundary $J$ is a simple closed curve. Suppose $X$ has a locally finite triangulation $T$. Let $S$ denote the subcomplex of $T$ consisting of the two-dimensional simplexes that intersect $J$ together with their faces (i.e., the star of $J$ in $T$ ). Then the complement of $|S|$ in $X$ has finitely many components $C_{0}, C_{1}, \ldots, C_{p}$ with closures $L_{0}, L_{1}, \ldots, L_{p}$, respectively, each bounded by a simple closed curve. Exactly one of the closures, say $L_{0}$, is noncompact; it is a half-open annulus containing a neighborhood of infinity in $X$. The others, $L_{1}, \ldots, L_{p}$, are disks. Define $A=X \backslash C_{0}$. Then $A$ is a well-triangulated annulus with islands. The original curve $J$ is the bottom boundary curve of $A$. The boundary curve of $L_{0}$ is the top boundary curve of $A$. The islands of A are those disks $L_{j}$ which contain a vertex of $T$ in their interior.

Lemma 21 (Open Disk Construction Lemma). In the statement of the Annulus Construction Lemma, replace $X$ by an open disk; replace its boundary $J$ by a single vertex of $T$; and replace $S$ by the star of the vertex $J$. Then $L_{0}$, the noncompact closure, is once again a half-open annulus. The set $A=X \backslash C_{0}$ is a disk.

Lemma 22 (Closed Disk Construction Lemma). In the statement of the Annulus Construction Lemma, replace $X$ by a closed disk, and replace $J$ by its boundary. Then all of the closed components $L_{1}, \ldots, L_{p}$, if any, are disks.

Note that we are reserving $L_{0}$ to refer to the unbounded components in the construction lemmas.

Lemma 23 (Addendum to Construction Lemmas). Let $L_{0}, L_{1}, \ldots, L_{p}$ be the closed components arising in any of the three construction lemmas. Form a graph $\Gamma$ as follows: put one vertex in the interior of each of the sets $L_{i}$; put one vertex at each vertex of $T$ which lies on any of the boundaries of the sets $L_{i}$; and join the interior vertex of $L_{i}$ to each of its boundary vertices by an edge. Then each component of $\Gamma$ is a tree.

Proof. Suppose one component of $\Gamma$ is not a tree. Then there is some simple closed curve $K$ in $\Gamma$. At least one of the vertices of $K$ must be in the interior of some $L_{k}$, implying that one of the points on the boundary of $L_{k}$ must be separated by $K$ from $J$. We may assume that $K$ is embedded in the union of the sets $L_{i}$. However, each boundary point of every $L_{i}$ can be joined to $J$ by an arc which lies (except for its endpoints) in the interior of some single triangle of $T$ which hits $J$. By definition, the union of the sets $L_{i}$ 
cannot contain this triangle, and thus we must conclude that none of these arcs hit $K$. This is a contradiction, and the result follows.

The following proposition is an immediate consequence of the first two construction lemmas:

Proposition 24. Let $G$ be a locally finite disk triangulation. Pick a base vertex $v_{0}$, and for each positive integer $k$ define $V_{k}=\{v|| v \mid=k\}$. Then there exist sets $\left\{A_{i}\right\}_{i=0}^{\infty}$ such that $G=\bigcup_{i=0}^{\infty} A_{i}$ and such that the following statements are true:

1. $A_{0}$ is a closed topological disk.

2. For $i \geq 1, A_{i}$ is a well-triangulated annulus with islands.

3. For $i \geq 1$, all bottom vertices of $A_{i}$ are in $V_{i}$; and all top vertices of $A_{i}$ are in $V_{i+1}$. Note that this statement implies that all islands in $A_{i}$ are stationed at $i$.

4. The bottom of $A_{i}$ is equal to the top of $A_{i-1}$ if $i \geq 2$, and the bottom of $A_{1}$ is equal to the boundary of $A_{0}$.

Proof. Let $G$ be a disk triangulation with a distinguished vertex $v_{0}$. Then, by the Open Disk Construction Lemma, there exists a closed disk $A_{0}$ containing the star of $v_{0}$, and $A_{0}$ has all of its boundary vertices in $V_{1}$. Note also that all of the vertices in $V_{1}$ are contained in $A_{0} . G \backslash A_{0}$ is a half-open annulus.

We have now proved the first conclusion of the proposition. We prove the rest of the conclusions inductively.

Suppose we have complexes $\left\{A_{i}\right\}_{i=0}^{k}$ with a finite number of vertices. Let $D_{k}=$ $\bigcup_{i=0}^{k} A_{i}$, and suppose that the following statements hold:

1. $G \backslash D_{k}$ is a half-open annulus.

2. The vertices on $\partial D_{k}$ are all in $V_{k+1}$.

3. All vertices of $\bigcup_{i=1}^{k+1} V_{i}$ are contained in $D_{k}$.

We refer to these statements as Induction Facts for the remainder of this proof. Clearly they are satisfied for $k=0$. Now suppose they are satisfied for some $k$.

Let $X_{k}$ be the half-open annulus $G \backslash D_{k}$ with boundary $\partial X_{k}=\partial D_{k}$. By the Annulus Construction Lemma we may obtain a well-triangulated annulus with islands $A_{k+1}$. This fact establishes the second conclusion of the proposition. Since $A_{k+1}$ contains the star of $\partial D_{k}$, we have established our first Induction Fact for $k+1$. The bottom boundary curve of $A_{k}$ will be $\partial D_{k}$ and thus will contain only vertices of $V_{k+1}$. Because of our construction, the top boundary of $A_{k+1}$ can only contain vertices of $V_{k+2}$, thus establishing the third conclusion of the proposition and our second Induction Fact for $k+1$. The fourth conclusion of the proposition also follows from our method of constructing $A_{k+1}$, as does our third Induction Fact. Hence we have completed our induction.

By our third Induction Fact every vertex of $G$ is contained in at least one set $A_{k}$, and hence we can decompose $G$ into the union of well-triangulated annuli with islands.

We use the following lemma to replace an island by a set of interior vertices. 
Lemma 25. Let $P$ be a graph homeomorphic to a circle. Suppose $P$ has $n$ vertices, labeled $v_{1}, \ldots, v_{n}$, with $n \geq 3$. Then there is a triangulation $T_{P}$ with the following properties:

1. $\left|T_{P}\right|$ is a closed topological disk.

2. $P$ is the boundary of $T_{P}$.

3. All vertices of $T_{P}$ lie on its boundary.

4. $\sum_{i=1}^{n}\left(\operatorname{tval}\left(v_{i}\right)-3\right)=-6$, where $\operatorname{tval}(\cdot)$ counts only triangles inside $T_{p}$.

Proof. In order to prove this lemma, we must triangulate the region bounded by $P$ without introducing any new vertices. To do so, note that there exists a vertex $v_{j}$ on $P$ such that $v_{j}$ is not the endpoint of an edge which both joins two vertices of $P$ and at the same time is exterior to the region bounded by $P$. Now cone from $v_{j}$, joining it to the other nonadjacent vertices of $P$, and the first three statements of the lemma are self-evident. To prove the last statement, note that if $T_{P}$ consists of a single triangle, the conclusion is obvious. Otherwise, apply the shelling lemma and then the uniform reduction procedure. One can then verify the final conclusion inductively via a simple calculation. (Note that only shelling disks of type one can arise, $T_{P}$ having no interior vertices.)

If $T_{P}$ is a triangulation with $n$ vertices as described in Lemma 25, we call it a pseudoisland of size $n$. We use pseudoislands to show that the presence of an island in a well-triangulated annulus with islands does not materially affect the sum of tile valences along the bottom.

Now we must prove some lemmas concerning the structure of islands themselves.

Lemma 26. Let $L$ be an island stationed at $k_{0}$. Suppose the island contains a vertex $v$ which is in the set $V_{n}$ for some $n \geq k_{0}+2$. Then $v$ is surrounded by a cycle of vertices, all of which lie in $V_{n-1}$. Furthermore, all vertices surrounded by this cycle will be in $\bigcup_{j=n}^{\infty} V_{j}$.

Proof. We prove the lemma by induction on $n$. By definition it is true for $n=k_{0}+2$. Assume the lemma is true for all vertices $v$ in $L$ such that $|v|=n$ for some $n \geq k_{0}+2$. Suppose now that we have a vertex $v$ in $L$ with $|v|=n+1$. Then there is an edge connecting $v$ to a vertex $w$ in $V_{n}$. By inductive hypothesis there is a cycle $C$ of vertices in $V_{n-1}$ surrounding $w$ and hence surrounding $v$ as well. Note that $|u| \geq n$ for all vertices $u$ enclosed by $C$.

Apply the Closed Disk Construction Lemma to the disk bounded by $C$. Then since $|v|=n+1, v$ cannot be in the star of $C$, all vertices of $C$ being in $V_{n-1}$. Hence it is enclosed by one of the disks $L_{i}$ referred to in that lemma; the boundary of $L_{i}$ consists of vertices in $V_{n}$, and $L_{i}$ contains only vertices $u$ such that $|u| \geq n+1$. Lemma 26 follows by induction for all values of $n \geq k_{0}+2$.

Lemma 27 builds on the construction lemmas to give details on the possible ways that islands may intersect. 
Lemma 27. Let $L_{0}, \ldots, L_{p}$ be the components referred to in any of the construction lemmas. The boundaries of the sets $L_{i}$ will be cycles of vertices. Then any two of these cycles intersect in at most one vertex, and there are in fact no cycles of cycles.

Proof. For each $i$, let $C_{i}=\partial L_{i}$. Since these cycles $C_{i}$ were formed by application of the construction lemmas, now consider the addendum to these lemmas. As described in that addendum, form a graph $\Gamma$ by putting one vertex in the interior of each cycle $C_{i}$ and connecting it to all boundary vertices of $C_{i}$. Then, according to the addendum, there are no cycles in $\Gamma$. Hence we conclude that the cycles $C_{i}$ can share at most one vertex per pair of cycles and that there are indeed no cycles of cycles.

Lemma 28. Let $L_{1}, \ldots, L_{p}$ be the closed and bounded components referred to in any of the construction lemmas. The boundaries of the sets $L_{i}$ will be cycles of vertices. For this lemma, let the term "interior vertex" denote any vertex in $\bigcup_{i=1}^{p} \partial L_{i}$ not lying on the original curve $J$ and not lying on the boundary of any unbounded $L_{0}$ that might exist. If $x^{L_{i}}$ is the number of vertices in $\partial L_{i}$, then the number of interior vertices in the union of the cycles is at least $\sum_{i=1}^{p} x^{L_{i}}-p$.

Proof. Once again form the graph $\Gamma$ as in the addendum to the construction lemmas. As usual, we procede by induction. First let $\Gamma_{1}=\Gamma \cap\left(L_{1} \cup L_{0}\right)$. Then the number of interior vertices on $\Gamma_{1}$ is at least $x^{L_{1}}-1$, since by Lemma $27 L_{1}$ can share at most one vertex with $L_{0}$ (assuming $L_{0}$ exists). Now assume that $\Gamma_{i}$ has been formed by the union of some subset of the collection of sets $\left\{\Gamma \cap L_{j}\right\}_{j=1}^{p}$. If not all of the sets $L_{j}$ have been used, choose one such $L_{j}$ to add to the union in order to form $\Gamma_{i+1}$. If possible, however, choose the $j$ such that $\Gamma \cap L_{j}$ intersects $\Gamma_{1}$. This subgraph $\Gamma \cap L_{j}$, according to Lemma 27, shares at most one vertex with $\Gamma_{i-1}$. Hence, the cycle $L_{j}$ must add at least $x^{L_{j}}-1$ vertices to the total, and the conclusion of the lemma follows.

Suppose a simplicial 2-complex is homeomorphic to a closed disk; suppose that it has vertices in its interior; and suppose that all interior vertices are connected to the boundary by an edge. We call such a complex an island core. We prove a fact about island cores before returning our attention to islands themselves.

Lemma 29. Suppose a triangulated disk $D$ has $x$ boundary vertices and $y$ interior vertices. Let $v_{1}, \ldots, v_{x}$ denote the boundary vertices, and let $w_{1}, \ldots, w_{y}$ denote the interior vertices. Then

1. $\sum_{i=1}^{x}\left(\operatorname{tval}\left(v_{i}\right)-3\right)+\sum_{i=1}^{y}\left(\operatorname{tval}\left(w_{i}\right)-6\right)=-6$;

2. $\sum_{i=1}^{x}\left(\operatorname{tval}\left(v_{i}\right)-3\right) \geq-3+y-x$.

Proof. Once again we use the shelling lemma and induction on the number of triangles. Both conclusions are clearly true for a single triangle. By the shelling lemma there exists a shelling disk $\Delta$ for $D$. Remove $\Delta$ via the uniform reduction procedure. A few easy calculations show that the lemma is inductively true.

Now is the best place to present a corollary to Lemma 25: 
Corollary 30. Let $L$ and $\bar{L}$ be an island and a pseudoisland, respectively, having the same boundary. Let $\operatorname{tval}(v)$ denote the tile valence of a vertex $v$ on the boundary of $L$, and let $\operatorname{tval}^{\prime}(v)$ denote the tile valence of the same vertex in $\bar{L}$. (In these tile valence numbers, count only the tiles inside $L$ or $\bar{L}$, respectively.) Then

$$
\sum_{v \in \partial(L)}\left(\operatorname{tval}(v)-\operatorname{tval}^{\prime}(v)\right)=6+\sum_{v \in \partial(L)}(\operatorname{tval}(v)-3) .
$$

Proof. Summing over the vertices $v$ in $\partial(L)$, we have

$$
\begin{aligned}
\sum_{v}\left(\operatorname{tval}(v)-\operatorname{tval}^{\prime}(v)\right) & =\sum_{v}(\operatorname{tval}(v)-3)-\sum_{v}\left(\operatorname{tval}^{\prime}(v)-3\right) \\
& =\sum_{v}(\operatorname{tval}(v)-3)+6
\end{aligned}
$$

by Lemma 25 .

In the proof of Theorem 6, we use Proposition 24 to partition the triangulated plane into a disk $A_{0}$ and annuli $A_{k}$. We also partition the valence sums $a_{k}=\sum_{|v| \leq k}(\operatorname{val}(v)-6)$ into contributions from the disk $A_{0}$ and the annuli $A_{1}, \ldots, A_{k}$.

We estimate the contribution from an annulus $A_{i}$ in two steps. We first replace islands by pseudoislands and calculate the contributions. Then Proposition 31, which follows, allows us to calculate the correction we must make in passing back to the original island.

For an interior vertex $v$ of an island, the adjustment is easy: simply add a summand equal to val $(v)-6$, namely, the contribution to valence excess which disappears when a pseudoisland (with no internal vertices) replaces the original island.

When $v$ is a boundary vertex of an island $L$, however, the matter is more subtle. It is tempting to think that the error involves how much was subtracted from val $(v)$, that is, that the error involves the 3 or the 6 that we subtract. On the contrary, the error resides in the valence term itself. One can decompose the valence term for $v$ as a sum. Let $\operatorname{tval}_{i}(v)$ denote the valence of $v$ in the island $L_{i}$. Let $\operatorname{tval}_{0}(v)$ denote the valence of $v$ in the complement of the islands of $A_{k}$. Then to find the total valence in an annulus $A_{k}$, calculate the sum $\sum_{i=0}^{p} \operatorname{tval}_{i}(v)$. The only correction that must be made is to replace the $\operatorname{tval}_{i}^{\prime}(v)$ as calculated in the pseudoisland with the $\operatorname{tval}_{i}(v)$ as calculated in the original island. Corollary 30 has already calculated the result.

Hence we may determine the total contribution of an island to the tile valence sums. If $L$ is an island stationed at $k_{0}$, then for all $k \geq k_{0}+1$, we will let $x_{k}^{L}$ be the number of vertices contained in $L$ that belong to the set $V_{k}$. If $k-k_{0}-1$ exceeds the depth of the island, then of course $x_{k}^{L}$ will be 0 .

Proposition 31. Let $L$ be an island stationed at $k_{0}$ with a depth of $n$. Define $a_{k}^{L}$ for all positive integers $k$ as follows:

$$
a_{k}^{L}= \begin{cases}0 & \text { if } \quad k \leq k_{0}, \\ 6+\sum_{|v|=k_{0}+1}(\operatorname{tval}(v)-3) & \text { if } \quad k=k_{0}+1, \\ a_{k_{0}+1}^{L}+\sum_{j=2}^{k-k_{0}} \sum_{|v|=k_{0}+j}(\operatorname{tval}(v)-6) & \text { if } \quad k \geq k_{0}+2,\end{cases}
$$


where the sums are taken only over the appropriate vertices in L. Then the following statements are true:

1. $a_{k}^{L}=0$ if $k \leq k_{0}$;

2. $a_{k}^{L}>x_{k+1}^{L}-x_{k}^{L}$ if $k_{0}+1 \leq k \leq k_{0}+n$;

3. $a_{k}^{L}=0$ if $k>k_{0}+n$.

Proof. The first conclusion is simply the definition of $a_{k}^{L}$ for $k \leq k_{0}$. To prove the rest, we perform induction on the depth of the island. First suppose that the depth is 1, i.e., the only interior vertices of the island are in $V_{k_{0}+2}$. Since the boundary vertices are the only vertices of $L$ which are in $V_{k_{0}+1}$, each of the interior vertices must be connected to a boundary vertex by an edge. Thus the island is an island core. Label the boundary vertices $v_{1}, \ldots, v_{x_{k_{0}+1}^{L}}$, and label the interior vertices $w_{1}, \ldots, w_{x_{k_{0}+2}^{L}}$.

$$
\begin{aligned}
a_{k_{0}+1}^{L} & =6+\sum_{i=1}^{x_{k_{0}+1}^{L}}\left(\operatorname{tval}\left(v_{i}\right)-3\right) \\
& >6-3+x_{k_{0}+2}^{L}-x_{k_{0}+1}^{L} \quad \text { (by Lemma 29) } \\
& >x_{k_{0}+2}^{L}-x_{k_{0}+1}^{L} .
\end{aligned}
$$

Also,

$$
\begin{aligned}
a_{k_{0}+2}^{L} & =a_{k_{0}+1}^{L}+\sum_{i=1}^{x_{k_{0}+2}^{L}}\left(\operatorname{tval}\left(w_{i}\right)-6\right) \\
& =6+\sum_{i=1}^{x_{k_{0}+1}^{L}}\left(\operatorname{tval}\left(v_{i}\right)-3\right)+\sum_{i=1}^{x_{k_{0}+2}^{L}}\left(\operatorname{tval}\left(w_{i}\right)-6\right) \\
& =6-6 \quad(\text { by Lemma } 29) .
\end{aligned}
$$

Clearly $a_{k_{0}+k}^{L}$ will be zero for $k>2$.

So we have proved the proposition for islands of depth 1 ; now we suppose we have proved it for islands of depth $n$. Let $L$ have a depth of $n+1$. By Lemma 26, all vertices in the island that belong to $V_{k_{0}+n+2}$ are encircled by a cycle of vertices (in the island) that belong to $V_{k_{0}+n+1}$; furthermore, we obtained these cycles by using the construction lemmas, and hence by Lemma 27 we may choose these cycles such that the interiors of the regions bounded by them are disjoint. Each of the vertices enclosed in these cycles must be in $V_{k_{0}+n+2}$ and hence have an edge connecting it to the cycle enclosing it; thus, each of these cycles is the boundary of an island core. Label these island cores $L_{1}, \ldots, L_{p}$. Replace each of them with a pseudoisland of the same size. We have now reduced the depth of the island by 1 ; call this reduced island $\bar{L}$. We know the following about $\bar{L}$ :

$$
\begin{aligned}
a_{k_{0}+1}^{\bar{L}} & >x_{k_{0}+2}^{L}-x_{k_{0}+1}^{L}, \\
& \vdots \\
a_{k_{0}+n}^{\bar{L}} & >x_{k_{0}+n+1}^{L}-x_{k_{0}+n}^{L}, \\
a_{k_{0}+n+1}^{L} & =0 .
\end{aligned}
$$


Observe that replacing the island cores by the pseudoislands did not affect any vertices in $V_{k_{0}+1}, \ldots, V_{k_{0}+n}$. We may thus say

$$
\begin{aligned}
a_{k_{0}+1}^{L} & >x_{k_{0}+2}^{L}-x_{k_{0}+1}^{L}, \\
& \vdots \\
a_{k_{0}+n}^{L} & >x_{k_{0}+n+1}^{L}-x_{k_{0}+n}^{L} .
\end{aligned}
$$

Now the difference between $a_{k_{0}+n+1}^{\bar{L}}$ and $a_{k_{0}+n+1}^{L}$ will be the difference between the tile valence sums on the interior of the pseudoislands and the tile valence sums on the interior of the island cores $L_{1}, \ldots, L_{p}$. We commit a slight abuse of terminology and notation by considering each of these island cores $L_{i}$ as an island stationed at $k_{0}+n$. Then the difference between the tile valence sum on the interior of $L_{i}$ and on the interior of the pseudoisland replacing it will be $a_{\left(k_{0}+n\right)+1}^{L_{i}}$. By Lemma 27 the interiors of the island cores $L_{i}$ are disjoint; hence, $\sum_{i=1}^{p} x_{k_{0}+n+2}^{L_{i}}=x_{k_{0}+n+2}^{L}$. By Lemma 28 we can see that $\sum_{i=1}^{p} x_{k_{0}+n+1}^{L_{i}} \leq x_{k_{0}+n+1}^{L}+p$.

Putting this together,

$$
\begin{aligned}
a_{k_{0}+n+1}^{L} & =a_{k_{0}+n+1}^{\bar{L}}+\sum_{i=1}^{p} a_{\left(k_{0}+n\right)+1}^{L_{i}} \\
& \geq 0+\sum_{i=1}^{p}\left(3+x_{k_{0}+n+2}^{L_{i}}-x_{k_{0}+n+1}^{L_{i}}\right) \quad \text { (by Lemma } 29 \text { and the proof above) } \\
& \geq 3 p+x_{k_{0}+n+2}^{L}-\left(x_{k_{0}+n+1}^{L}+p\right) \\
& >x_{k_{0}+n+2}^{L}-x_{k_{0}+n+1}^{L} .
\end{aligned}
$$

Finally, note that

$$
a_{k_{0}+n+1}^{L}=a_{k_{0}+n+1}^{\bar{L}}+\sum_{i=1}^{p} a_{\left(k_{0}+n\right)+1}^{L_{i}}=0+\sum_{i=1}^{p}\left(6+\sum_{\substack{v \in L_{i} \\|v|=k_{0}+n+1}}(\operatorname{tval}(v)-3)\right)
$$

and that

$$
\sum_{\substack{w \in L \\|w|=k_{0}+n+2}}(\operatorname{tval}(w)-6)=\sum_{i=1}^{p} \sum_{\substack{w \in L_{i} \\|w|=k_{0}+n+2}}(\operatorname{tval}(w)-6) .
$$

Thus we may say

$$
\begin{aligned}
a_{k_{0}+n+2}^{L} & =a_{k_{0}+n+1}^{L}+\sum_{i=1}^{x_{k_{0}+n+2}^{L}}\left(\operatorname{tval}\left(w_{i}\right)-6\right) \\
& =6 p+\sum_{i=1}^{p} \sum_{\substack{v \in L_{i} \\
|v|=k_{0}+n+1}}(\operatorname{tval}(v)-3)+\sum_{i=1}^{p} \sum_{\substack{w \in L_{i} \\
|w|=k_{0}+n+2}}(\operatorname{tval}(w)-6) \\
& =6 p-6 p \quad \text { (by Lemma 29). }
\end{aligned}
$$


Since $L$ only has a depth of $n+1, a_{k}^{L}$ will be zero for all values of $k$ greater than $k_{0}+n+2$, and thus we have proved the proposition.

We later have occasion to sum the numbers $a_{k}^{L}$. Hence we state the following corollary:

Corollary 32. Let $L$ be an island stationed at $k_{0}$ with a depth of $n$. Then for all positive values of $k$,

$$
\sum_{j=1}^{k} a_{j}^{L} \geq-x_{k_{0}+1}^{L}+1
$$

Proof. We show that $\sum_{j=1}^{k} a_{j}^{L}>-x_{k_{0}+1}^{L}$. By Proposition 31 we know that

$$
\begin{array}{ll}
a_{k}^{L}=0 & \text { for } \quad k \leq k_{0}, \\
a_{k}^{L}>x_{k+1}^{L}-x_{k}^{L} & \text { for } \quad k_{0}+1 \leq k \leq k_{0}+n, \\
a_{k}^{L}=0 & \text { for } \quad k_{0}+n<k .
\end{array}
$$

If $k \leq k_{0}$, then clearly $\sum_{j=1}^{k} a_{j}^{L}=0>-x_{k_{0}+1}^{L}$, and

$$
\sum_{j=1}^{k_{0}+1} a_{j}^{L}=\sum_{j=1}^{k_{0}} a_{j}^{L}+a_{k_{0}+1}^{L}>0+\left(x_{k_{0}+2}^{L}-x_{k_{0}+1}^{L}\right) .
$$

We now use induction. Suppose we have shown that, for some $k$, such that $k_{0}+1 \leq$ $k<k_{0}+n$, we have $\sum_{j=1}^{k} a_{j}^{L}>x_{k+1}^{L}-x_{k_{0}+1}^{L}$. Then, for $k+1$, we have

$$
\begin{aligned}
\sum_{j=1}^{k+1} a_{j}^{L} & =\sum_{j=1}^{k} a_{j}^{L}+a_{k+1}^{L} \\
& >\left(x_{k+1}^{L}-x_{k_{0}+1}^{L}\right)+\left(x_{k+2}^{L}-x_{k+1}^{L}\right) \\
& =x_{k+2}^{L}-x_{k_{0}+1}^{L} .
\end{aligned}
$$

This induction shows that for all $k$ such that $k_{0}+1 \leq k \leq k_{0}+n$, we have $\sum_{j=1}^{k} a_{j}^{L}>$ $x_{k+1}^{L}-x_{k_{0}+1}^{L}$, and hence $\sum_{j=1}^{k} a_{j}^{L}>-x_{k_{0}+1}^{L}$.

Finally, it is clear from Proposition 31 that for any $k>k_{0}+n$,

$$
\sum_{j=1}^{k} a_{j}^{L}=\sum_{j=1}^{k_{0}+n} a_{j}^{L}>-x_{k_{0}+1}^{L} .
$$

Now we want to show that the presence of islands in a well-triangulated annulus with islands cannot decrease its modulus.

Proposition 33. Let $X$ be a well-triangulated annulus with islands. Suppose $X$ has $n$ top vertices, and let $M$ be the fat flow modulus of $X$. Then

$$
M \geq \frac{3}{2 n} \text {. }
$$


Proof. Suppose $w$ is a vertex on the boundary of an island, and suppose $w$ does not lie on the top of $X$. We know that $w$ is connected to a bottom vertex $v$ by an edge. As we have done before, replace $v$ by two bottom vertices $v^{\prime}$ and $v^{\prime \prime}$, and replace $w$ by a bottom vertex $w^{\prime}$, whose neighbors on the bottom to either side are $v^{\prime}$ and $v^{\prime \prime}$.

Perform this operation on every vertex that does not lie on the top of $X$ but which is on the boundary of an island. The result will be a well-triangulated annulus $X_{0}^{\prime}$, possibly still with islands. Note that the number of top vertices of $X_{0}^{\prime}$ is still $n$. Any island in $X$ has become either a region with all of its boundary vertices on the bottom, or a region with some boundary vertices on the top and the rest on the bottom. We consider these two cases separately.

Let $M_{0}$ be the fat flow modulus of $X_{0}^{\prime}$. How does $M_{0}$ compare with $M$, the modulus of $X$ ? Let $w_{0}$ be an optimal fat flow weight function for $X_{0}^{\prime}$. Since every tile in $X_{0}^{\prime}$ corresponds to a tile in $X$, we can apply the function $w_{0}$ to the original tiling $X$. By the correspondence of the tiles, $A_{w_{0}}(X)=A_{w_{0}}\left(X_{0}^{\prime}\right)$. Note that every fat flow in $X_{0}^{\prime}$ is a subset of a fat flow in $X$, and every fat flow in $X$ contains a fat flow in $X_{0}^{\prime}$. Therefore, $H_{w_{0}}(X) \geq H_{w_{0}}\left(X_{0}^{\prime}\right)$. Thus, we have

$$
M \geq \frac{H_{w_{0}}^{2}(X)}{A_{w_{0}}(X)} \geq \frac{H_{w_{0}}^{2}\left(X_{0}^{\prime}\right)}{A_{w_{0}}\left(X_{0}^{\prime}\right)}=M_{0} .
$$

Now we consider the two cases. In the first case all boundary vertices are on the bottom. Since the region of the former island is still bounded by a cycle of vertices, the leftmost and rightmost boundary vertices must be connected by a single edge lying in the interior of $X_{0}^{\prime}$. We remove all tiles lying between the bottom and this edge (see Fig. 11), thus discarding all tiles that originally belonged to the island. In general, if the tiling was called $X_{n}^{\prime}$ before the operation, then we call the tiling resulting from the operation $X_{n+1}^{\prime}$. Note that $X_{n+1}^{\prime}$ has the same number of top vertices as $X_{n}^{\prime}$.

How does the modulus of $X_{n+1}^{\prime}$ compare with the modulus of $X_{n}^{\prime}$ ? Let $w_{n+1}$ be an optimal weight function on $X_{n+1}^{\prime}$. Extend $w_{n+1}$ to $X_{n}^{\prime}$ by letting $w_{n+1}(t)=0$ for all tiles in $X_{n}^{\prime} \backslash X_{n+1}^{\prime}$. Clearly $H_{w_{n+1}}\left(X_{n}^{\prime}\right)=H_{w_{n+1}}\left(X_{n+1}^{\prime}\right)$ and $A_{w_{n+1}}\left(X_{n}^{\prime}\right)=A_{w_{n+1}}\left(X_{n+1}^{\prime}\right)$. Therefore, if $M_{n+1}$ is the fat flow modulus of $X_{n+1}^{\prime}$ and $M_{n}$ is the fat flow modulus of $X_{n}^{\prime}$, we have

$$
M_{n+1}=\frac{H_{w_{n+1}}^{2}\left(X_{n+1}^{\prime}\right)}{A_{w_{n+1}}\left(X_{n+1}^{\prime}\right)}=\frac{H_{w_{n+1}}^{2}\left(X_{n}^{\prime}\right)}{A_{w_{n+1}}\left(X_{n}^{\prime}\right)} \leq M_{n} .
$$

Consider the second case, in which some boundary vertices are on the top. Suppose that $t_{1}, \ldots, t_{n}$ are the tiles in the island that are adjacent to the top boundary vertices.

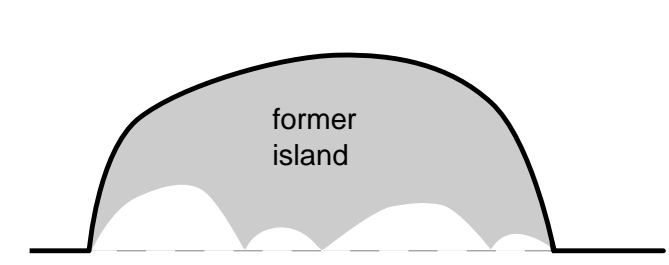

Fig. 11. Removing an island when no island vertices were on the top. 


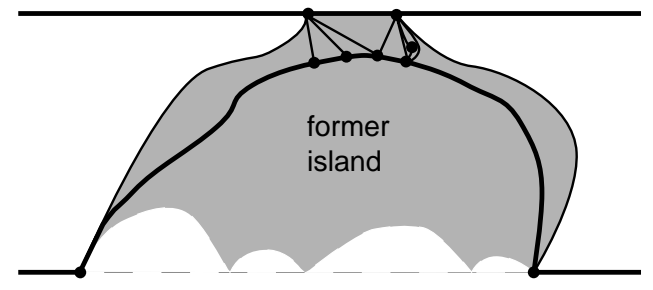

Fig. 12. Removing an island when some island vertices were on the top.

We remove all tiles lying between the tiles $t_{1}, \ldots, t_{n}$ and the bottom. (See Fig. 12.) Note that islands might remain in place after this operation, coming from tiles inside the original island. As before, if the tiling before the operation was $X_{n}^{\prime}$, then the tiling after the operation will be called $X_{n+1}^{\prime}$. Note that $X_{n+1}^{\prime}$ has the same number of top vertices as $A_{n}^{\prime}$. The same argument as in the previous case shows us that $M_{n+1} \leq M_{n}$.

Since there can only be a finite number of tiles in $X$, we may perform this operation until we obtain a well-triangulated annulus (without islands) $X_{q}^{\prime}(q \geq 0)$ having the same number of top vertices $n$ as does $X$. By Proposition $19 M_{q} \geq 3 /(2 n)$. Hence, we may conclude that

$$
M \geq M_{0} \geq M_{1} \geq \cdots \geq M_{q} \geq \frac{3}{2 n}
$$

At last we are in a position to see that islands will not affect the parabolicity of a disk triangulation graph, thus proving Theorem 6.

Theorem 6. Let $G$ be a disk triangulation graph of bounded valence. Let $v_{0}$ be a vertex of $G$. For any vertex of $G$, let $|v|$ denote the minimum number of edges in a path connecting $v$ to $v_{0}$. Let

$$
a_{n}=\sum_{|v| \leq n}(\operatorname{val}(v)-6) .
$$

If the sequence $\left\{a_{n}\right\}$ is bounded, then $G$ is parabolic.

Proof. Let $\left\{A_{j}\right\}_{j=0}^{\infty}$ be the decomposition of $G$ given by Proposition 24. Recall that $A_{0}$ is a closed topological disk and each $A_{j}$ (for $j \geq 1$ ) is a well-triangulated annulus with islands such that the bottom vertices are in $V_{j}$ and the top vertices are in $V_{j+1}$.

We first remove all islands from $G$ and calculate the corresponding valence sums; afterwards we take islands into account.

Let $\bar{G}$ be the graph $G$ altered by replacing each island (in the concentric welltriangulated annuli with islands $A_{i}$ ) with a pseudoisland of the same size. Each annulus $A_{j}$ in our decomposition is thereby replaced with a well-triangulated annulus $\bar{A}_{j}$ in $\bar{G}$ such that all bottom vertices of $\bar{A}_{j}$ are in $V_{j}$ and all top vertices are in $V_{j+1}$.

In the graph $\bar{G}$ we define

$$
\bar{a}_{k}=\sum_{\substack{v \in \bar{G} \\|v| \leq k}}(\operatorname{val}(v)-6) .
$$

We use the decomposition $\left\{\bar{A}_{j}\right\}$ of $\bar{G}$ to partition the valence excess sums $\bar{a}_{k}$ as follows. 
For each nonnegative integer $k$, let

$$
J_{k}=\sum_{\substack{v \in \tilde{A}_{0} \\|v| \leq k}}(\operatorname{val}(v)-6) .
$$

Note that the integers $J_{k}$ do not change once $k$ is so large that $\bar{A}_{0}$ contains no vertices from $V_{k}$. Thus we may choose a positive integer $J$ larger than all of the integers $-J_{k}$, where $k=0,1,2, \ldots$ The integer $J$ allows us to bound the contributions of the disk $\bar{A}_{0}=A_{0}$ to the valence excess sums $\bar{a}_{k}$.

Each annulus $\bar{A}_{k}$ has sets $B_{k}, T_{k}$, and $I_{k}$ of bottom, top, and interior vertices, respectively, which contribute to the sums $\bar{a}_{k}$. Denote the number of vertices in each set by $c_{k}=\left|B_{k}\right|, n_{k}=\left|T_{k}\right|$, and $x_{k}=\left|I_{k}\right|$. Now define $b_{k}=\sum_{B_{k}}(\operatorname{tval}(v)-3)$; define $t_{k}=\sum_{T_{k}}(\operatorname{tval}(v)-3)$; and define $i_{k}=\sum_{I_{k}}(\operatorname{tval}(v)-6)$. The tile valence counts on $B_{k}$ and $T_{k}$, of course, take into account only tiles in $\bar{A}_{k}$.

Taking into account that the boundary vertices of $\bar{A}_{0}$ serve as bottom vertices of $\bar{A}_{1}$ and that, for $k>0$, the top vertices of $\bar{A}_{k}$ serve as bottom vertices of $\bar{A}_{k+1}$, we obtain the following equality:

$$
\bar{a}_{k}=\left(J_{k}-b_{1}\right)+\sum_{j=1}^{k-1}\left(b_{j}+i_{j}+t_{j}\right)+b_{k}=J_{k}-b_{1}+b_{k},
$$

where $b_{j}+i_{j}+t_{j}=0$ by Proposition 11 since $\bar{A}_{j}$ is a well-triangulated annulus without islands.

Now we take the islands into account. We combine the numbers $\bar{a}_{k}$ above and the numbers $a_{k}^{L}$ of Proposition 31 to estimate the original sums in $G$, namely,

$$
a_{k}=\sum_{\substack{v \in G \\|v| \leq k}}(\operatorname{val}(v)-6) .
$$

The logic of forming the sum $a_{k}$ from $\bar{a}_{k}$ and the island numbers $a_{k}^{L}$ is as follows. Fix $k \geq 2$. Begin with $\bar{G}$. Note that $\bar{a}_{k}$ contains the summands val $(v)-6$ for the vertices $v$ of $\bar{A}_{0}=A_{0}$ such that $|v| \leq k$, as well as for all vertices $v$ of $\bar{A}_{1}, \ldots, \bar{A}_{k-1}$. Note that there can only be two factors contributing to the differences between $\bar{a}_{k}$ and $a_{k}$ :

1. A vertex $v$ of $G$ such that $|v| \leq k$ lies in the interior of an island $L$ of $A_{1}, \ldots, A_{k-1}$ and hence does not appear in $\bar{G}$.

2. A vertex $v$ of $G$ such that $|v| \leq k$ lies on the boundary cycle of an island of $A_{1}, \ldots, A_{k-1}$ but does not have the same valence in $G$ as it had in $\bar{G}$.

However, as noted in Corollary 30 and in the statement, proof, and discussion of Proposition 31 , adding $\sum\left\{a_{k}^{L} \mid \operatorname{dist}\left(\partial L, v_{0}\right) \leq k\right\}$ to $\bar{a}_{k}$ precisely corrects both defects. Hence we have the precise equality

$$
a_{k}=\bar{a}_{k}+\sum_{\operatorname{dist}\left(\partial L, v_{0}\right) \leq k} a_{k}^{L}=\left(J_{k}-b_{1}\right)+b_{k}+\sum_{\operatorname{dist}\left(\partial L, v_{0}\right) \leq k} a_{k}^{L} .
$$


If $p_{k}$ is the number of islands containing vertices $v$ such that $|v| \leq k$, we may index the islands $L$ as $L_{1}, \ldots, L_{p_{k}}$ and write

$$
a_{k}=\left(J_{k}-b_{1}\right)+b_{k}+\sum_{i=1}^{p_{k}} a_{k}^{L_{i}} .
$$

By hypothesis, the sequence $\left\{a_{k}\right\}$ of valence excess numbers is bounded. Thus there exists a $B$ such that $a_{k}<B$ for all $k$; hence, by Proposition 10, we know that

$$
\left(J_{k}-b_{1}\right)+n_{k}+x_{k}-c_{k}+\sum_{i=1}^{p_{k}} a_{k}^{L_{i}} \leq\left(J_{k}-b_{1}\right)+b_{k}+\sum_{i=1}^{p_{k}} a_{k}^{L_{i}}=a_{k}<B,
$$

and hence

$$
\begin{aligned}
n_{k} & <B+c_{k}-x_{k}-\sum_{i=1}^{p_{k}} a_{k}^{L_{i}}-J_{k}+b_{1} \\
& \leq\left(B+J+b_{1}\right)+c_{k}-\left(x_{k}+\sum_{i=1}^{p_{k}} a_{k}^{L_{i}}\right) .
\end{aligned}
$$

We do some more induction. We know

$$
n_{1} \leq\left(B+J+b_{1}\right)+c_{1}-\left(x_{1}+\sum_{i=1}^{p_{1}} a_{1}^{L_{i}}\right) .
$$

Suppose, for some $l<k$,

$$
n_{l} \leq l\left(B+J+b_{1}\right)+c_{1}-\left(\sum_{j=1}^{l} x_{j}+\sum_{j=1}^{l} \sum_{i=1}^{p_{j}} a_{k}^{L_{i}}\right) .
$$

Then, by (1),

$$
\begin{aligned}
n_{l+1} & \leq\left(B+J+b_{1}\right)+c_{l+1}-x_{l+1}-\sum_{i=1}^{p_{l+1}} a_{l+1}^{L_{i}} \\
& =\left(B+J+b_{1}\right)+n_{l}-x_{l+1}-\sum_{i=1}^{p_{l+1}} a_{l+1}^{L_{i}} \quad\left(\text { since } c_{l+1}=n_{l}\right) \\
& \leq(l+1)\left(B+J+b_{1}\right)+c_{1}-\left(\sum_{j=1}^{l+1} x_{j}+\sum_{j=1}^{l+1} \sum_{i=1}^{p_{j}} a_{j}^{L_{i}}\right)
\end{aligned}
$$

by (2). Thus, for $k$ in particular,

$$
n_{k} \leq k\left(B+J+b_{1}\right)+c_{1}-\left(\sum_{j=1}^{k} x_{j}+\sum_{j=1}^{k} \sum_{i=1}^{p_{j}} a_{j}^{L_{i}}\right) .
$$


Now, since $a_{k}^{L}=0$ if $L$ contains no vertices of $V_{k}$,

$$
\begin{aligned}
\sum_{j=1}^{k} \sum_{i=1}^{p_{j}} a_{j}^{L_{i}} & =\sum_{j=1}^{k} \sum_{i=1}^{p_{k}} a_{j}^{L_{i}} \\
& =\sum_{i=1}^{p_{k}} \sum_{j=1}^{k} a_{j}^{L_{i}} \\
& \geq-\sum_{i=1}^{p_{k}} x_{s_{i}+1}^{L_{i}}+p_{k}
\end{aligned}
$$

by Corollary 32, where $s_{i}$ is the station of $L_{i}$.

Lemma 28 asserts that there are at least $\sum_{i=1}^{p_{k}} x_{s_{i}+1}^{L_{i}}-p_{k}$ interior vertices. Hence we have

$$
-\sum_{j=1}^{k} \sum_{i=1}^{p_{j}} a_{j}^{L_{i}} \leq \sum_{i=1}^{p_{k}} x_{s_{i}+1}^{L_{i}}-p_{k} \leq \sum_{j=1}^{k} x_{j} .
$$

So

$$
\sum_{j=1}^{k} x_{j}+\sum_{j=1}^{k} \sum_{i=1}^{p_{j}} a_{j}^{L_{i}} \geq 0
$$

and we conclude that

$$
n_{k} \leq k\left(B+J+b_{1}\right)+c_{1} .
$$

This inequality is true for all values of $k$. However, each $A_{k}$ is a well-triangulated annulus with islands. Therefore, by Proposition 33, if $M_{k}$ is the modulus of $A_{k}$, then

$$
M_{k} \geq \frac{3}{2 n_{k}} \geq \frac{3}{2\left(k\left(B+J+b_{1}\right)+c_{1}\right)} .
$$

Let $M$ be the fat flow modulus of $G$. Then, by the Layer Theorem,

$$
\begin{aligned}
M & \geq \sum_{k=1}^{\infty} M_{k} \\
& \geq \frac{3}{2} \sum_{k=1}^{\infty} \frac{1}{k\left(B+J+b_{1}\right)+c_{1}} \\
& =\infty .
\end{aligned}
$$

\section{Acknowledgments}

Many thanks are due to Bill Floyd, who suggested many of the foundational ideas in this paper to me. The anonymous referees also deserve credit for many helpful suggestions, especially concerning improvement of the sections involving valence counts and disk or annulus constructions. 


\section{References}

1. J. W. Cannon, The Combinatorial Riemann Mapping Theorem, Acta Mathematica, 173 (1994), 155-234.

2. J. W. Cannon, W. J. Floyd, and W. R. Parry, Conformal Modulus: The Graph Paper Invariant, or The Conformal Shape of an Algorithm, in Geometric Group Theory Down Under, de Gruyter, Berlin, 1999, pp. 71-102.

3. J. W. Cannon, W. J. Floyd, and W. R. Parry, On the Conformal Invariance of Tiling Systems, preprint, 1996.

4. J. W. Cannon, W. J. Floyd, and W. R. Parry, Squaring Rectangles: The Finite Riemann Mapping Theorem, Contemporary Mathematics, 169 (1994), 133-212.

5. J. W. Cannon, W. J. Floyd, and W. R. Parry, The Length-Area Method and Discrete Riemann Mappings, preprint, based on a talk given by J. W. Cannon at the Ahlfors Celebration, Stanford University, Stanford, CA, 1997.

6. Z.-X. He and O. Schramm, Hyperbolic and Parabolic Packings, Discrete \& Computational Geometry, 14 (1995), 123-149.

7. P. Pansu, Dimension conforme et sphère à l'infini des variétés à courbure négative. Annales Academiae Scientiarum Fennicae. Series AI. Mathematica, 14 (1989), 177-212.

8. A. S. Repp, Discrete Riemann Maps and the Parabolicity of Tilings, Ph.D. thesis, Virginia Tech, Blacksburg, VA, 1998.

9. O. Schramm, Square Tilings with Prescribed Combinatorics, Israel Journal of Mathematics, 84 (1993): 97-118.

Received April 14, 1999, and in revised form June 21, 2000. Online publication August 28, 2001. 